\title{
Mini Review: Some Peculiarities of Chemical Mesoscopics and This Scientific Trend Development Perspective
}

\author{
Kodolov Vladimir Ivanovitch ${ }^{1,2}$, Kodolova-Chukhontzeva Vera Vladimirovna ${ }^{1,3}$, \\ Mustakimov Rostislav Valer'evitch ${ }^{1}$ \\ ${ }^{1}$ Mesocomposites Department, High-Educational Centre of Chemical Physics \& Mesoscopics, Udmurt Federal Research Centre, Russian \\ Academy of Sciences, Izhevsk, Russia \\ ${ }^{2}$ Department of Chemical Technology, V. T. Kalashnikov Izhevsk State Technical University, Izhevsk, Russia \\ ${ }^{3}$ Research Laboratory "Polymeric Materials for Tissue Engineering and Transplantation", Peter the Great St. Petersburg Polytechnic \\ University St. Petersburg, Russia
}

\section{Email address:}

vikodolov@mail.ru (K. V. Ivanovitch),kodol@istu.ru (K. V. Ivanovitch)

\section{To cite this article:}

Kodolov Vladimir Ivanovitch, Kodolova-Chukhontzeva Vera Vladimirovna, Mustakimov Rostislav Valer'evitch. Mini Review: Some Peculiarities of Chemical Mesoscopics and This Scientific Trend Development Perspective. Science Journal of Chemistry.

Vol. 9, No. 4, 2021, pp. 97-104. doi: 10.11648/j.sjc.20210904.12

Received: July 5, 2021; Accepted: July 19, 2021; Published: August 26, 2021

\begin{abstract}
In present mini review the definition of new scientific trend "chemical mesoscopics", which is based on the ideas of the mesoscopic physics (mesoscopics) and also more latest mesoscopic chemistry, is given. This new scientific trend includes quantum notions on mesoparticle reactivity in chemical reactions. In these cases the negative charges quants interactions (interference) are considered as well as the interactions of negative and positive charges (annihilation). The schemes of the chemical bonds formation at the reactions flowing into mesoscopic reactors are presented. The especial attention is devoted to the interactions of metal (Copper, Nickel) carbon mesoparticles with such oxidizers as Ammonium Polyphosphate and Aluminum Oxide. In these reactions it is observed the reduction of Phosphorus and Aluminum, the growth of mesocomposite metal atomic magnetic moment and the simultaneous increasing of spin quantity on the mesocomposite carbon shell. The introduction of "annihilation" notion explains the increasing of magnetic characteristics. The modification of polymeric materials (on example of epoxy resins) by metal carbon mesocomposites is considered. For the mesoparticle (nanostructures) reactivity estimation the proposals are done. These proposals are concluded in the using of Fractal Theory ideas (Kolmogorov - Avrami equations) together with energetic analogs of reactivity theory.
\end{abstract}

Keywords: Chemical Reactions, Charge Quantization, Interference, Annihilation, Nanostructures Reactivity, Red Ox Reactions, Atomic Magnetic Moments, Kolmogorov-Avrami Equations Changed

\section{Introduction}

Chemical Mesoscopics as new trend in Chemical Sciences was appeared from such scientific trends as Synergetics (Self Organization), Fractal Theory (Self Similarity), Theories of Chemical Kinetics and Catalysis [1-3].

All of these trends are used for the description of mesoscopic particles (or nanostructures) behavior in the different media and at the various conditions changes. Therefore the Mesoscopic Physics and later appeared Mesoscopic Chemistry can be presented as the basis of
Chemical Mesoscopics. Above new trend is very near to Chemical Physics on the considered objects and also on the phenomena and particularities of the various reactions and processes at the changes of conditions of them realization.

However, the basic aim of this advanced trend is appeared the investigation of nanostructures (or mesoparticles) reactivity in the various media and at the different changed conditions. 


\section{Chemical Mesoscopics Ideas in the Chemical Reactions of Material Obtaining}

According to known notions the reactions can be divided into reactions realized without the elements oxidation states changes and reduction oxidation reactions in which the change of oxidation states for elements participated in process take place.

The reactions which flow without of oxidation states changes for elements participating in the processes are concerned to the chemical bonds formation or its destruction.

The chemical bonds formation owing to the electron wave's interference phenomenon was proposed by K. Ruedenberg in his book "Physical Nature of Chemical Bond" [4].

According to Ruedenberg, the bond energy $\left(E_{b}\right)$ can be expressed by three energetic components (equation 1) -

$$
E_{b}=E^{0}+E^{\prime}+E^{\prime}
$$

where $E^{0}$ - Coulomb component, E' - interference energy component, E" - energy component which is concerned to the energy of interaction between the different bonds and which is near to zero because of various directions of interactions. The last energy component can be not taken into account on above reason.

In the reactions which flow without the changes of elements oxidation states the hybridization phenomena take place at the negative charges quants radiation (or electromagnetic radiation). The correspondent radiation can be single measured, two or three measured that can be compared with the hybridization types as $\mathrm{sp}, \mathrm{sp}^{2}$ and $\mathrm{sp}^{3}$ and, consequently, with the molecules forms. This phenomena influence on polarization of the correspondent substances and media. The hybridization phenomena and the changes of electron structure are investigated by the main method of Chemical Mesoscopics $-\mathrm{x}$ ray photoelectron spectroscopy. This method is also used for the determination of substances nature as well as metal atomic magnetic moments.

In the practice it's possible that the chemical reactions flow in the mesoscopic (nanosized) reactors or multi plates which are described as the space between the same banks (may be parts of reagent or catalyst molecules). Mechanism based on the Chemical Mesoscopics notions for electron transport across mesoparticle (for example, metal cluster) from one to other bank (mesoscopic reactor walls) is proposed (Figure 1).

$$
\begin{aligned}
& \mu 1=\mu+\mathrm{e} \varphi 1 \\
& \text { I I I I I I I bank } 1 \\
& \Delta \mu \uparrow \uparrow e \text { o } \uparrow \text { e } \uparrow \text { e } \\
& \begin{array}{lllllll}
1 & 1 & 1 & 1 & \perp & \perp
\end{array} \\
& \mu 2=\mu+\mathrm{e} \varphi 2 \\
& \mathrm{M}(\mathrm{n}+)+\mathrm{ne} \rightarrow \mathrm{M}
\end{aligned}
$$

Figure 1. Scheme of electron transport and the Red Ox reactions within mesoscopic reactors [5].
At the reduction oxidation reaction the annihilation phenomenon is appeared. The process of annihilation creation is occurred by the following actions: the negative charge quants are directed to positive charged atom, near nucleus which the positive charge quants are located, and interact with them. As a result, the annihilation with the electromagnetic direct field formation takes place. This field stimulates the electron shift with the activation of negative charge quants and then the interference of these quants (electron waves) with the new chemical bonds formation. Thus, the reduction oxidation processes can be explained by two phenomena: annihilation and interference following one after another.

The introduction of the correspondent mesoparticles in media or compositions leads to the polarization of them. Therefore the modification conditions for the different materials can be differed one from another.

According to scheme of possible polarization the increasing of medium (material) density owing to the regular orientation of material fragments with the creation of super molecular and crystalline structures takes place (Figure 2).

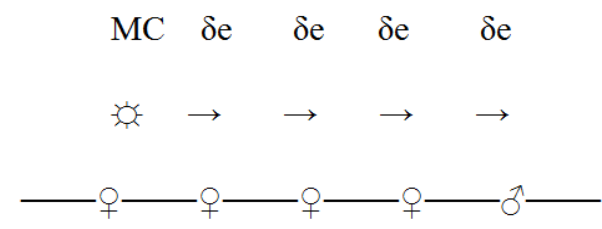

Figure 2. Scheme of polarization at charge quantization with expansion of quant influence on materials polar groups.

Designations: MC ( (electron), $\rightarrow$ the polarization direction, $q-q-$ macromolecule fragment with functional groups.

Polarization growth can be expressed as $\mathrm{P}_{\text {com }}=\Sigma \mathrm{p}_{\mathrm{fg}}+\mathrm{p}_{\mathrm{NC}}$, (2)

where $\mathrm{P}_{\text {com }}$ - the common (summary) polarization, $\Sigma \mathrm{p}_{\mathrm{fg}}-$ sum of functional groups polarizations, $\mathrm{p}_{\mathrm{NC}}-$ the polarization (or dipole moment) of mesocomposite.

The polarization extent depends on the quants electromagnetic radiation phase velocity. It's necessary to note that this velocity will be decreased in the media with high dielectric constant according to following formula -

$$
\mathrm{v}=\mathrm{c} / \sqrt{ } \varepsilon
$$

where $\mathrm{v}$ - the phase velocity of electromagnetic radiation, $\mathrm{c}-$ the light velocity, $\varepsilon-$ dielectric constant.

When the dielectric constant is increased the decrease of mesocomposite influence on the media arises and the self organization process is finished.

Depending on the development of self organization process (single measured - 1D, double measured - 2D, third measured - 3D) the super molecular structures (mesoparticles) of correspondent forms and sizes are organized. The surface energy of embryos increased influences on the mesoparticles formation. This energy can be express as the sum of energetic components for the realization of different movements: 


$$
\mathrm{E}_{\mathrm{S}}=\mathrm{E}_{(\mathrm{rr})}+\mathrm{E}_{(\mathrm{r})}+\mathrm{E}_{(\mathrm{osc})}+\mathrm{E}_{(\mathrm{em})},
$$

where $E_{S}$ - surface energy of macromolecule (mesoparticle), $E_{(t r)}$ - part of translational motion energy, $E_{(r)}$ - part of rotary motion energy, $\mathrm{E}_{(\mathrm{osc})}$ - part of oscillatory motion energy, $\mathrm{E}_{(\mathrm{em})}$ - part of electron motion in surface layer.

In accordance with the formation of mesoparticles which have the identical orientation with each other, the parts of translational motion energy and of rotary motion energy will be near to zero $[5,6]$. Therefore the main contribution into the mesoparticle surface energy will be bring the oscillatory motion and transport of electrons in surface layer of macromolecules (mesoparticles). Then the change of character of quants radiation wave propagation from 2D (in the surface plane) to $3 \mathrm{D}$ (in the space field at surface) takes place.

\section{The Confirmations of Above Notions on the Examples of the Metal Carbon Mesoscopic Composites Modification}

The above ideas are considered on the examples of the metal carbon mesoscopic composites modification reactions and also different polymeric materials. The hypothesis about possibility of annihilation at the interaction of positive and negative charges quants in red ox processes is comfirmed by the examples of processes of Copper and Nickel Carbon mesocomposites modification with application such substances as polyethylene polyamine, ammonium iodide, ammonium polyphosphate (APP), silica $\left(\mathrm{SiO}_{2}\right)$, aluminium oxide, iron oxide, nickel oxide and copper oxide [7-9]. In the case, when polyethylene polyamine and ammonium iodide are applied, the connection reactions takes place. At the interactions of polyethylene polyamine with mesoparticles the $\mathrm{C}=\mathrm{N}$ bond formation is explained by the interference of negative charges quants. When the mesoparticles modification reactions with the using $\mathrm{APP}, \mathrm{SiO}_{2}$, metal oxides are carried out, the redox processes are realized. In these cases the modifiers reduction reactions take place. The structures of metal carbon mesoscopic composites with active carbon shells are defined by means of the complex of methods including x-ray photoelectron spectroscopy, transition electron microscopy with high permission, electron microdiffraction and also EPR spectroscopy.

Below in tables 1 and 2 the examples of metal atomic magnetic moments changes (in Boron magnetons) and quantities of unpaired electrons (in spin/g) for mesoparticles modified by $\mathrm{APPh}$ or silica after the mechanochemical modification processes proceeding are given.

Table 1. The values of Copper (Nickel) atomic magnetic moments in the interaction products for systems: $\mathrm{Cu}-\mathrm{C} \mathrm{MC}-\mathrm{APPh}\left(\mathrm{or} \mathrm{SiO} \mathrm{O}_{2}\right.$ and $\mathrm{Ni-C} \mathrm{MC-APPh}$ $\left(\right.$ or $\mathrm{SiO}_{2}$ ).

\begin{tabular}{|c|c|c|c|}
\hline Systems Cu-C MC - substances & $\mu_{\mathrm{cu}}$ & Systems Ni-C MC - substance & $\mu_{\mathrm{Ni}}$ \\
\hline $\mathrm{Cu}-\mathrm{C} \mathrm{MC}$ - silica & 3,0 & Ni-C MC - silica & 4,0 \\
\hline $\mathrm{Cu}-\mathrm{C} \mathrm{MC}-\mathrm{APPh}$ & 2,0 & $\mathrm{Ni}-\mathrm{C} \mathrm{MC}-\mathrm{APPh}$ & 3,0 \\
\hline $\mathrm{Cu}-\mathrm{C} \mathrm{MC}-\mathrm{APPh}$, relation $1: 0,5$ & 4,2 & & \\
\hline
\end{tabular}

Table 2. The unpaired electron values (from EPR spectra) for systems " $\mathrm{Cu}-\mathrm{C}$ $M C-$ silica" and "Cu-C MC - APPh" (relation 1:1) in comparison with initial mesoparticle $\mathrm{Cu}$-C MC.

\begin{tabular}{ll}
\hline Substance & Quantity of unpaired electrons, spin/g \\
\hline $\mathrm{Cu}-\mathrm{C}$ mesocomposite & $1,2 \times 10^{17}$ \\
system «Cu-C $\mathrm{MC}-\mathrm{SiO}_{2} »$ & $3,4 \times 10^{19}$ \\
system «Cu-C $\mathrm{MC}-\mathrm{APPh} »$ & $2,8 \times 10^{18}$ \\
\hline
\end{tabular}

The metal atomic magnetic moment growth proceeds owing to the redox processes with above chemical compounds. In papers [7, 8] it's shown that the reduction reactions of Phosphorus and Silicon from correspondent substances at the interaction on the interphase boundary with mesoparticles are realized (Figures 3-5).

The increase of the metal atomic magnetic moment during the mixing of the $\mathrm{Cu}-\mathrm{C}$ mesocomposite with chemical compounds and the change of the number of delocalized electrons on carbon structures is connected with the interaction of a nanosized particle with the environment. The assumed mechanism of the increase of the metal atomic magnetic moment is the electron exchange at the phase boundary similar to heterogeneous catalysis in multi cells. For a large particle, the relation of the surface to mass is so small that the metal surrounding cannot influence the electron density of its d-zone; in this case, the magnetic moment of a small particle can noticeably change. It is shown in [8] that as a result of mechanochemical mixing, in the obtained systems "Cu-C - ammonium polyphosphate" and "Cu-C - silica", phosphorus or silicon of the compound are reduced; this fact confirms the interaction presence of mesocomposite with reagents on the phase boundary.

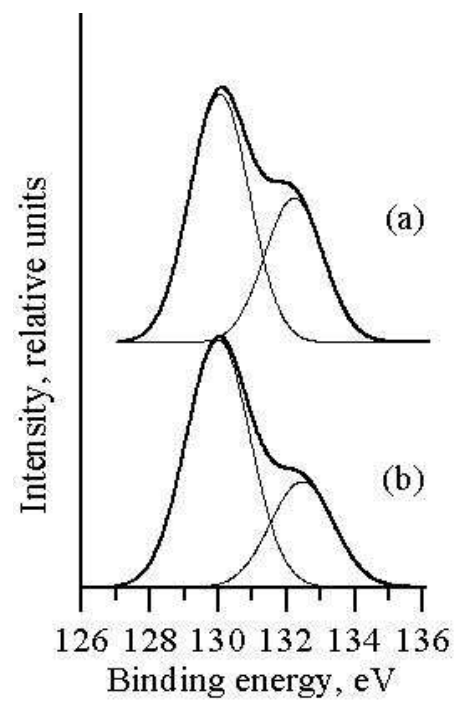

Figure 3. The XPS P2p spectra: a) $C u / C+A P P$ 1:1; b) $C u / C+A P P$ 1:0.5 [8]. 
The XPS P2p spectra for phosphorus-containing mesocomposites prepared at the ratio of presents the reagents $\mathrm{Cu}-\mathrm{C}$ : ammonium polyphosphate $=1$ and $\mathrm{Cu}-\mathrm{C}$ : ammonium polyphosphate $=2$ show on Figure 3 . The phosphorus reduction at the ratio of 2 is almost $50 \%$ more complete $[7,8]$ and from the comparison Figure $3 \mathrm{a}$ and Figure $3 \mathrm{~b}$.

Such result can be explained by the rate of the electron quantization because of the increasing the ammonium polyphosphate layer thickness.

In this case the structure Phosphorus containing Copper Carbon mesocomposite is similar to the initial mesocomposite, only the increasing of carbon fibers thickness is noted because of the possible disposition of phosphorus reduced between carbon atoms of carbon fibers.

On the Figure 4 the comparison of TEM microphotographs for the initial Copper Carbon mesoscopic composite and the Phosphorus containing this mesocomposite after modification by Ammonium Polyphosphate is given.

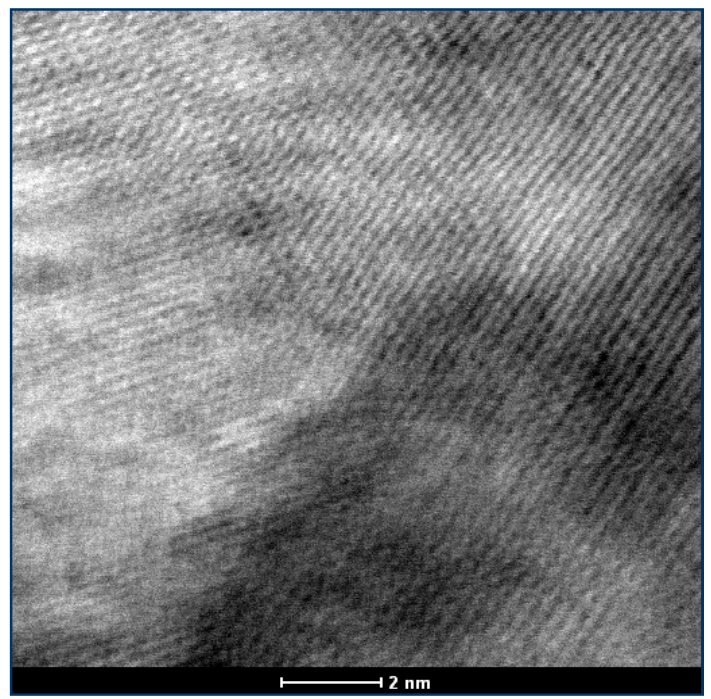

a

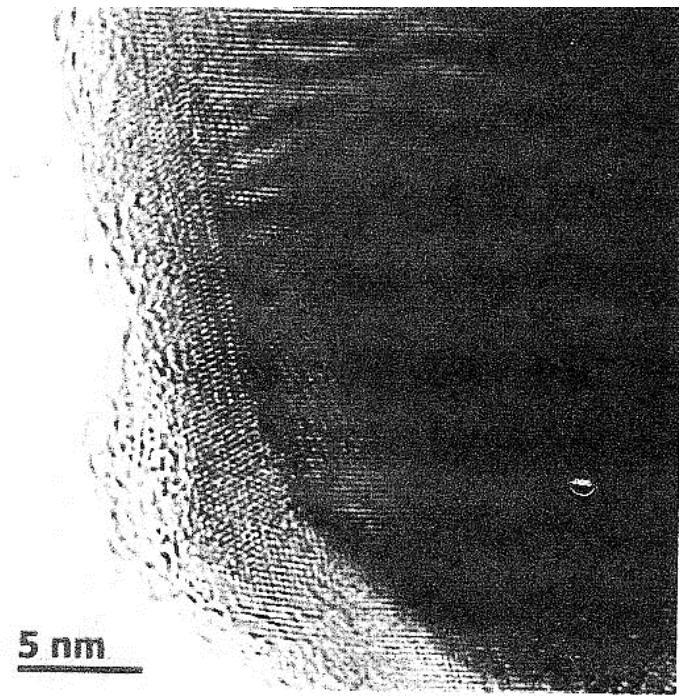

b

Figure 4. Comporison of TEM microphotographs for intial Copper Carbon mesocomposite (a) and Phosphorus containing $\mathrm{Cu} C$ mesocomposite (b).
The Phosphorus disposition between carbon fibers can be explained by the interaction of Phosphorus electrons with the carbine double bonds electrons that is confirmed by further studies on the reactions of mesocomposite obtained with different reagents.

At the mechanochemical processes of Ammonium Polyphosphate with Copper Carbon mesoscopic composite the ammoniac molecules are chipped off and evaporated. Therefore there is not nitrogen in $\mathrm{x}$-ray photoelectron spectrum of the product obtained. The presence of oxidized forms of Carbon and Phosphorus is determined by the results of x-ray photoelectron spectroscopy and electron sound micro roentgen spectroscopic analysis of element composition. The results obtained attest about the addition of acidic P-O-H groups, which are formed after the ammoniac evaporation, to the double bonds of carbon envelope with the formation $\mathrm{C}-\mathrm{H}$ and $\mathrm{P}-\mathrm{O}-\mathrm{C}$ bonds. According to $\mathrm{x}$-ray photoelectron spectra the binding energy equaled to $133 \mathrm{eV}$ corresponds to P-O bond, and the correspondent energy equaled to $286 \mathrm{eV}-\mathrm{C}-\mathrm{O}$ bond. In all probability the phosphorus is disposed between the carbon fibers of $\mathrm{Cu}-\mathrm{C}$ mesoscopic carbon shell and is reduced because of the interaction with unpaired electrons which are found on the mesocomposite carbon shell. The reduction process may be presented by the following multi-stage scheme: the first stage - the negative charges quants are directed to the positive charged nucleus of Phosphorus atom, near which there is the cloud of positive charge quants; the second stage- the interaction of negative charged quants with positive charged quants, which leads to the annihilation phenomena creation; the third stage - at the annihilation the inner electromagnetic field is formed which stimulates the Phosphorus reduction process; the fourth stage - at the reduction process the water vapors are formed and are evaporated, that leads to $\mathrm{C}=\mathrm{P}$ bond formation $(132,6 \mathrm{eV}$ on $\mathrm{x}$-ray photoelectron spectra); the fifth stage - the electro magnetic radiation formed stimulates across the coordination bonds $d$ orbital of Copper atom that leads to the formation of unpaired electrons and the growth of Copper atomic magnetic moment; the sixth stage- the development of fifth stage and explains the increasing of the spins number on the carbon shell of Copper cluster.

The similar explanation of reduction oxidation reactions mechanism is possible at the interaction of Aluminum oxide with Copper Carbon mesoscopic composite. In this case Aluminum is reduced completely with simultaneous growth of Copper atomic magnetic moment from 1,3 to 4,8 Bohr magnetons. These changes are corresponded with the increasing of spin number on the Carbon shell from $10^{17}$ $\mathrm{spin} / \mathrm{g}$ to $10^{19} \mathrm{spin} / \mathrm{g}[10]$.

Thus, it's possible to change the magnetic properties of mesoparticles and the number of unpaired electrons using the Red Ox reactions of metal carbon mesocomposites with different oxidizers.

In the case, when ammonium polyphosphate is replaced by aerosol (or silicon), the reduction of silicon from silicon oxide takes place by $50 \%$ independent of the used amount of the reagents (Figure 5); this can be explained by the decrease 
of the rate of the electron quantization in the layer containing Si-O bonds.

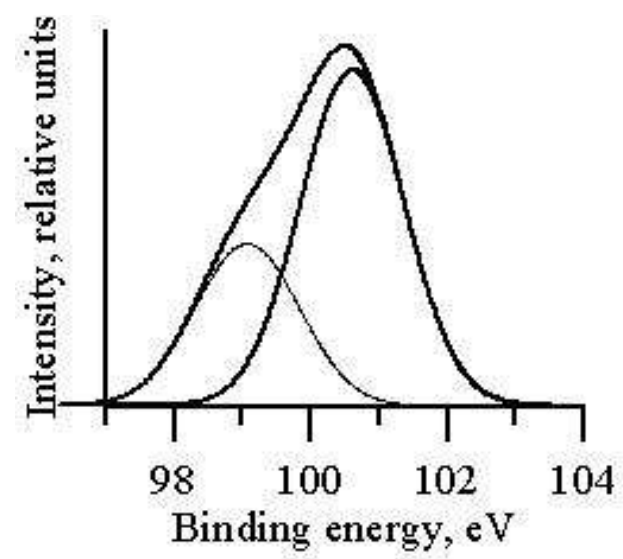

Figure 5. The XPS Si2p spectrum for the sample $\mathrm{Cu} / \mathrm{C}+\mathrm{SiO}_{2}$ at the ratio $1: 1(E(\mathrm{SiO})=100.7 \mathrm{eV} ; \mathrm{E}(\mathrm{Si})=99 \mathrm{eV})$.

The early created method $[10,11]$ on the base of the x-ray photoelectron spectra is used for the Copper Carbon mesocomposite modification mechanism investigation. The inner levels spectra for $\mathrm{C} 1 \mathrm{~s}, \mathrm{O} 1 \mathrm{~s}, \mathrm{Si} 2 \mathrm{p}, \mathrm{P} 2 \mathrm{p}, \mathrm{S} 2 \mathrm{p} \mathrm{Cu} 3 \mathrm{~s}$ are studied to investigate the mechanism of chemical bonds formation between metal or carbon atoms and silicon or phosphorus atoms. It's noted the presence of changes in the $\mathrm{Cu} 3 \mathrm{~s}$ spectra in mesocomposites modified in comparison with initial mesocomposite.

\section{The Influence of Metal Carbon Mesocomposites on the Polymeric Substances Modification Processes}

The media (or polymeric compositions) properties changes under the mesoparticles influence can be achieved at equal distribution of these particles in composition volume and at its coagulation absence. Last is possible at the following conditions:

1) certain polarity and dielectric constant of medium;
2) minute concentration of mesoparticles;

3) Ultrasound action on the correspondent suspension for the proportional distribution of mesoparticles.

The assignment of active nanostructures (mesoparticles) during the compositions modification is concluded in the activation of matrices self organization in needful direction. For the realization of this goal the determination of organized phase part is necessary.

In some papers [12-20] the positive results on materials properties improvement are presented when the minute quantities of metal carbon mesocomposites are introduced in these materials. In paper [17] the hypothesis about nanostructures influence transmission on macromolecules of polymeric matrices is proposed. This hypothesis is complied with mesoscopic physics principles which consider quantum effects at the certain conditions of mesoparticle existence.

The composition polarization is possible because of there is the charge quantization with the wave expansion on polar (functional) groups of media (for example, polymer macromolecule). The quantum charge wave expansion leads to the functional groups polarization (dipole moments) change as well as the extinction increasing. Last bring growth of peaks intensities in IR spectra. The individual peaks growth effects in IR spectra are observed at the introduction of mesocomposites minute quantities (Table 3). It's necessary to note, that the peaks intensity growth in IR spectra is observed when the quantity of introduced mesocomposite is decreased. This fact is complied with fundamental principles of chemical mesoscopic. In illustrated case the instance of fine dispersed suspension $\mathrm{Cu}-\mathrm{C}$ mesocomposite (hardener for epoxy resin). According to data of Table 3 the decreasing of mesocomposite quantity to $0,001 \%$ leads to the growth of some peaks intensity in IR spectra.

At the second day of that suspension existence the floccules are formed and peaks intensity sharply drops. However the suspension activity can be increased with the using of ultrasound treatment. The treatment optimal duration determined as 7 minutes. In this case the IR spectra peak intensity in is increased in 2-4 times (Table 4).

Table 3. The peaks intensity change in dependence of $\mathrm{Cu}$-C mesocomposite concentration.

\begin{tabular}{|c|c|c|c|c|c|}
\hline $\mathbf{N}$ & $\mathrm{v}, \mathrm{cm}^{-1}$ & $\mathbf{I}_{1} / \mathbf{I}_{0}$ & $\mathbf{I}_{0,01} / \mathbf{I}_{0}$ & $\mathbf{I}_{0,001}$ & Chemical Bonds/Groups \\
\hline 1 & 1050 & 1,235 & 1,411 & 1,686 & $\mathrm{C}-\mathrm{O}-\mathrm{C}$ st \\
\hline 2 & 1450 & 1,179 & 1,590 & 1,744 & $\mathrm{C}-\mathrm{H}$ \\
\hline 3 & 1776 & 1,458 & 1,347 & 1,691 & $\mathrm{C}=\mathrm{O}$ st as \\
\hline 4 & 1844 & 1,463 & 1,412 & 1,678 & $\mathrm{C}=\mathrm{O}$ st sy \\
\hline 5 & $2860-3090$ & 1,182 & 1,545 & 1,750 & $\mathrm{C}-\mathrm{H}$ \\
\hline
\end{tabular}

Table 4. The peaks intensity changes in IR spectrum of Cu-C mesocomposite depending on the duration of ultrasound treatment.

\begin{tabular}{|c|c|c|c|c|}
\hline $\mathbf{N}$ & $\mathbf{v}, \mathrm{cm}^{-1}$ & $\mathbf{I}_{7} / \mathbf{I}_{0}$ & $\mathbf{I}_{10} / \mathbf{I}_{0}$ & Chemical Bonds/Groups \\
\hline 1 & 1776 & 3,7932 & 0,7574 & $\mathrm{C}=\mathrm{O}$ st as \\
\hline 2 & 1844 & 2,5065 & 0,9115 & $\mathrm{C}=\mathrm{O}$ st sy \\
\hline 3 & 3039 & 2,3849 & 0,9589 & C-H \\
\hline
\end{tabular}

The charge (electron) quantization should lead to the macromolecule electron structure change and, as corollary, to change sub molecular structures of polymeric substances.
In the monograph [6] it's established that the quants electron wave which initiates self organization process in polymeric composition is expensed from carbon fibers of 
cover associated with metal cluster in the metal carbon mesoscopic composite. The last leads to the correspondent orientation of sub molecular structures in nanostructured composite surface layers.

The self organization mechanism for polymeric compositions modified by the Metal Carbon mesocomposite minute quantities is concluded in the conditions creation for the composition polarization, which bring the great change of electron and sub molecular structures of materials.

In this case it's possible the following explanation: with the mesoparticle quantity decreasing the creation of diffused radiation of electron quants directed to polymeric polar functional groups is possible that leads to $\mathrm{sp}^{3}$ hybridization and 3D form of polymer surface layer. In other words, it's possible there is necessity to take into account the forms of quants flows directed to active groups of polymers modified. Certainly, these changes influence on the modified materials properties.

\section{The Proposal for the Estimation of Nanostructures Reactivity Estimation}

According to the fractal theory [3] any system can be presented as aggregate of elements similar to whole system. These elements have own energetic and geometric (volume) parameters owing to which they are found within the system. The change of these parameters because of the action of external factors leads to disturbance of system balance. At this case the system is destructed or transformed. The estimation of these changes is possible with the using of the relative parameters in which the energetic and volume values are compared with the definite standard values for the correspondent elements (fragments) in the definite reaction series. This approach to reactivity consideration is near to Taft and Pal'm theoretical works [23]. For the relative energetic parameters the following formula $\left(\varepsilon-\varepsilon_{0}\right) / \varepsilon_{0}$ is proposed [24], where $\varepsilon_{0}$ corresponds to the surface energy for standard chemical fragment. In turn, analogous relative parameters are proposed [25] for the volume characteristics $\left(\mathrm{V}-\mathrm{V}_{0}\right) / \mathrm{V}_{0}$.

The development of Chemical Mesoscopics in this direction is connected with the research of the size and energetic characteristics of chemical particles. The size of mesoparticles is denoted as approximately $10 \mathrm{~nm}$, and the motion freedom of nanostructures (mesoparticles) is limited by the vibration with high frequency and electron transport across them. The peculiarities of mesoparticles consist in the radiation of energy quants of negative or positive charges. This radiation is the main reason of the stimulation of chemical processes. At the imposition of the negative charge quants the interference takes place and the chemical bonds are formed. In turn the imposition of the negative and positive quants together the phenomenon of annihilation is created. At this case the direct electromagnetic field is appeared that leads to the stimulation of negative charge quants moving and the growth of chemical bonds formation.
The phenomena of interference and annihilation are reasons of start for self organization process with reservation conformation order, which determine the finished product structure. For the process explanation the equation of Kolmogorov-Avrami [5] can be used

$$
\mathrm{W}=1-\exp \left(-\mathrm{k} \tau^{\mathrm{n}}\right)
$$

where $\mathrm{W}$ - the part of obtained product (for instance, polymer), $\mathrm{k}$ - the process rate constant, $\tau$ - the duration of process, $\mathrm{n}-$ the fractal dimension (for one measured process $\mathrm{n}=1)$.

For the comparative estimation of reagents (or nanostructures) in one reaction series it's possible the application of the theory of free energy linear dependences. In this case the reactions are considered with using one of reagent as the standard compound for which $\mathrm{W}$ is fixed $\mathrm{W}_{0}$. The estimation of reactivity can be proposed on the difference $\mathrm{W}-\mathrm{W}_{0}$, where $\mathrm{W}$ is calculated on formula 2 , and $\mathrm{W}_{0}$ is defined on analogous formula with changes $\mathrm{k}_{0} \tau_{0}{ }^{\mathrm{n}}$. It's noted, the fractal dimension $\mathrm{n}$ do not change because the comparison is carried out for one type of reactions. The following equation for difference $\mathrm{W}-\mathrm{W}_{0}$ can be written -

$$
\mathrm{W}-\mathrm{W}_{0}=\exp \left(-\mathrm{k}_{0} \tau_{0}\right)-\exp \left(-\mathrm{k} \tau^{\mathrm{n}}\right)
$$

and after eq. 2 transformation -

$$
\mathrm{W}_{0}=\mathrm{k} / \mathrm{k}_{0}\left(\tau / \tau_{0}\right)^{\mathrm{n}}-1
$$

If $\operatorname{lg~} \mathrm{k} / \mathrm{k}_{0}$ is defined as -

$$
\lg \mathrm{k} / \mathrm{k}_{0}=-2,3 \text { RT }\left\{\left[\left(\varepsilon_{0}-\varepsilon\right) / \varepsilon_{0}\right] a+\left[\left(\mathrm{V}_{0}-\mathrm{V}\right) / \mathrm{V}_{0}\right] b\right\},
$$

and then this expression after transformation stand in the eq. 3 , than the equation 5 is received -

$$
\lg \left(\mathrm{W}-\mathrm{W}_{0}\right)=\left(\tau / \tau_{0}\right)^{\mathrm{n}} \exp \left\{\left[\left(\varepsilon_{0}-\varepsilon\right) / \varepsilon_{0}\right] a+\left[\left(\mathrm{V}_{0}-\mathrm{V}\right) / \mathrm{V}_{0}\right] b\right\},
$$

where values $\mathrm{a}$ and $\mathrm{b}$ - parameters, which correct the influence polar and steric (spaced) effects on reactivity in polymerization, the relation $\left(\varepsilon_{0}-\varepsilon\right) / \varepsilon_{0} \quad[24,25]$ correspondents to Taft constant $\sigma$ (polarity constant), and the relation $\left(\mathrm{V}_{0}-\mathrm{V}\right) / \mathrm{V}_{0}-$ Taft constant $\mathrm{E}_{\mathrm{s}}$ (steric or spaced constant) [25].

The application of above notions for the Chemical Mesoscopics development is very perspective because stimulates the mathematic apparatus creation for the chemical processes flowing direction prediction.

\section{Conclusion}

On the base of presented materials it is possible to make the following conclusions:

1. In mini review the peculiarities of new scientific trend "chemical mesoscopics" at the mesoparticles reactivity description in their modification processes as well as in the polymeric compounds modification with the mesoparticles using are considered. The schemes of reactions mechanisms into mesoscopic reactors and also the mechanisms of media (compositions) polarization 
under the flow action of negative charges quants directed from the nanostructures or mesoparticles are discussed.

2. For the explanation of the chemical bonds formation in the reactions without the oxidation state change the notion "interference" is introduced, and for the explanation of reduction oxidation (Red-Ox) reactions mechanisms the notion "annihilation" is introduced. The application of these notions is considered on the examples of the metal carbon mesocomposites modification by the different substances, including oxidizers. With the "annihilation" notion application the oxidizer reduction as well as the mesocomposite metal atomic magnetic moment growth and also the spin quantity increasing on mesocomposite carbon shell are explained.

3. On the base of experimental results the scheme of mechanism of Copper Carbon mesocomposite modification by Ammonium Polyphosphate is given. In this process the Phosphorus is reduced and is disposed between the carbon fibers of mesoparticle carbon shell. The process is accompanied by the growth of Copper atomic magnetic moment from 1,3 to $4,2 \mathrm{Bohr}$ magnetons (at the reagents relation equaled to 2). Simultaneously it is noted the spin increasing in ten times on the mesoscopic carbon shell. Analogous results are observed at the interaction this mesocomposite with Aluminum Oxide.

4. The experimental results concerning to the epoxy resin suspension modification by the minute quantities of Copper Carbon mesocomposites and their modified compounds with IR spectroscopic investigations using are discussed. The minute quantities nanostructures (mesoparticles) application at the polymeric substances modification is substantiated.

5. The nanostructures (mesoparticles) reactivity estimation is proposed with the using Kolmogorov-Avrami equations and the energetic characteristics (polarity and volume or spatial constants) for the fragments of mesoscopic compounds.

The development new scientific trend "chemical mesoscopics" supposes the theory development for mechanisms of mesoscopic systems formation as well as mechanisms of self organization in media and compositions. The peculiarities of structures and energetic characteristics of mesocomposites obtained cause their possibilities for applications in the different fields.

The examples of applications in radical, red ox and addition processes as catalysts, reagents and also inhibitors as well as additives and modifiers improving properties of materials (inorganic and organic polymeric materials), adhesives and glues, fire proof systems, corrosion inhibitors, medicine magnetic transport remedies, stimulators of plant growth are presented in the review [5]. The Metal Carbon mesoscopic composites owing to their magnetic characteristics can be used in the electromagnetic radiation focal systems.

This unique scientific trend discover new era in the development of new theories in the natural sciences and in the practice, for instance, novel nanostructures application widening.

\section{References}

[1] Kodolov V. I., Trineeva V. V. New scientific trend - Chemical Mesoscopics - Chemical Physics \& Mesoscopics, 2017. V. 19. N 3. Pp. $454-465$.

[2] Kodolov V. I., Kodolova-Chukhontzeva V. V., Terebova N. S., Shabanova I. N. The explanation of magnetic metal carbon mesocomposites synthesis peculiarities by means of mesoscopic notions. - Academ. J. Polym. Sci. 2019, 3 (2): 555613.

[3] Moskaletz M. V. Fundamentals of Mesoscopic Physics. Khar'kov: NTU KhPI, 2010 - 180p.

[4] Ruedenberg K. Physical Nature of Chemical Bond. - M.: Publ "Mir", 1964 - 162p.

[5] Kodolov V. I., Kodolova-Chukhotzeva V. V., Shabanova I. N. et al. Review: Possible Fields of metal carbon mesocomposites application. - In Book: Innovation and Challenges in Modern Physical Chemistry. Research and Practice. - N. Y.: Nova Sciences Publishers, Inc., 2020, 195p. - Pp. 57-122.

[6] Kodolov V. I., Trineeva V. V., Pershin Yu. V. et al. Method of metal carbon nanocomposites obtaining from metal oxides and polyvinyl alcohol. -Pat. RU 2018122 001, 2020.

[7] Kodolov V. I., Kodolova -Chukhontseva V. V. Fundamentals of Chemical Mesoscopics. - Monograph. - Izhevsk: Publisher - M. T. Kalashnikov Izhevsk State Technical University, 2019 $-218 p$.

[8] Mustakimov R. V., Kodolov V. I., Shabanova I. N., Terebova N. S. Modification of copper carbon nanocomposites with the using of Ammonium Polyphosphate for the application as modifiers of epoxy resins.// Chemical Physics \& Mesoscopics, 2017. V. 19. N 1. Pp. 50-57.

[9] Kodolov V. I., Trineeva V. V., Kopylova A. A et al. Mechanochemical modification of metal carbon nanocomposites. // Chemical Physics \& Mesoscopics, 2017. V. 19. N 4. Pp. 569-580.

[10] Kodolov V. I., Trineeva V. V., Terebova N. S. et al. The change of electron structure and magnetic characteristics of modified copper carbon nanocomposites.// Chemical Physics \& Mesoscopics, 2018. V. 20. N 1. Pp. 72-79.

[11] Shabanova I. N., Kodolov V. I., Terebova N. S., Trineeva V. V. (2012) X ray electro spectroscopy in investigation of metal/carbon nanosystems and nanostructured materials. (P. 252) Izhevsk-Moscow: Publ. "Udmurt University".

[12] Shabanova I. N., Terebova N. S. Kodolov V. I. et al. (2013) The investigation of metal or carbon nanocomposites electron structure by $\mathrm{X}$ ray photoelectron spectroscopy. in Book: Nanostructure, nanosystems and nanostructured materials. Theory, production and development (Pp. 177-230). Toronto, Canada - New Jersey, USA: Apple Academic Press.

[13] Kodolov V. I., Lipanov A. M., Trineeva V. V. et al. (2013) The changes of properties of materials modified by metal/carbon nanocomposites. Ibid. (Pp. 327 - 373). 
[14] Kodolov V. I., Trineeva V. V. (2013) Theory of modification of polymeric materials by super small quantities of metal/carbon nanocomposites.// Chemical Physics \& Mesoscopy, V. 15. N 3. Pp. $351-363$.

[15] Kodolov V. I., Trineeva V. V. (2012). Perspectives of idea development about nanosystems self organization in polymeric matrixes In book "The problems of nanochemistry for the creation of new materials" - Torun, Poland: IEPMD. (Pp. 75-100).

[16] Akhmetshina L. F., Lebedeva G. A., Kodolov V. I. (2012). Phosphorus containing metal/carbon nanocomposites and their application for the modification of intumescent fireproof coatings. // Journal of Characterization and Development of Novel Materials, 2012. V. 4. N 4. Pp. 451-468.

[17] Kodolov V. I., Kovyazina O. A., Trineeva V. V., Vasilchenko Yu. M., Vakhrushina M. A., Chmutin I. A. On the production of metal/carbon nanocomposites, water and organic suspensions on their basis.// VII International ScientificTechnical Conference "Nanotechnologies to the production 2010"./ Proceedings. Fryazino, 2010. Pp. 52-53.

[18] Chashkin M. A. Kodolov V. I., Trineeva V. V. Metal/Carbon nanocomposites- Epoxy compositions Quantum Chemical Investigation and Experimental Modeling. // Polymers Research Journal, 2011. V. 5. N 1. Pp. 5 - 19.

[19] Patent 2337062 Russia Technique of obtaining carbon nanostructures from organic compounds and metal containing substances / Kodolov V. I., Kodolova V. V. (Trineeva), Semakina N. V., Yakovlev G. I., Volkova E. G. et al; declared on 28.08.2006, published on 27.10.2008.

[20] Kodolov V. I., Trineeva V. V., Kovyazina O. A., Vasilchenko Yu. M. Production and application of metal/carbon nanocomposites.// In book "The problems of nanochemistry for the creation of new materials" - Torun, Poland: IEPMD, 2012. Pp. $23-36$.

[21] Akhmetshina L. F., Kodolov V. I., Tereshkin I. P., Korotin A. I. The influence of carbon metal containing nanostructures on strength properties of concrete composites. // Internet Journal "Nanotechnologies in Construction", 2010. N 6. Pp. 35-46.

[22] Pershin Yu. V., Kodolov V. I. Polycarbonate modified with $\mathrm{Cu}-\mathrm{C}$ nanocomposite.//in Book "The problems of nanochemistry for the creation of new materials" - Poland Torun: Publ. IEPMD, 2012. - 250p. - Pp. $173-179$.

[23] V. A. Pal'm. The introduction in theoretical organic chemistry. - M.: Publ. "High School", 1974 - 446p.

[24] V. I. Kodolov. Possibilities of modeling in organic chemistry.// Organic Reactivity, 1965. V. 2. Iss. 4 (6). Pp. 11-17.

[25] V. I. Kodolov, S. S. Spasskiy. Parameters in Alfrey-Price and Taft equations. //Vysokomol. Soed., 1976. V. 18A. N 9. Pp. 1986-1992. 